Eur. J. Clin. Chem. Clin. Biochem.

Vol. 31, 1993, pp. 41-45

(C) 1993 Walter de Gruyter \& Co.

Berlin - New York

\title{
A HPLC-Based Chloramphenicol Acetyltransferase Assay for Assessing Hair Growth: \\ Comparison of the Sensitivity of UV and Fluorescence Detection
}

\author{
By D. J. Waldon, M. F. Kubicek, G. A. Johnson and A. E. Buhl \\ Dermatology Department, The Upjohn Company, Kalamazoo, MI USA
}

(Received June 22/October 6, 1992)

Summary: In our attempt to measure hair growth by hair-specific markers, we used transgenic mice to express the chloramphenicol acetyltransferase gene under the control of an ultrahigh sulphur keratin gene promoter. To quantitate expression of the keratin gene, we required a chloramphenicol acetyltransferase assay which could measure enzyme activity in a single follicle and also could be used to assay a large number of samples without loss of sensitivity. We achieved this objective by utilizing a fluorescent substrate for chloramphenicol acetyltransferase. With HPLC-fluorescence detection, this substrate provides a sensitivity of less than $1 \times 10^{-13} \mathrm{~mol}$, which is 1000 times greater than that achievable with HPLC-UV detection in cultured follicles. Further, the assay was automated to facilitate the analysis of more than 100 samples/day. It should be possible to apply this fluorescent assay to a number of cell or tissue studies.

\section{Introduction}

Assessment of hair growth or the measurement of end points related to hair growth is difficult due to the very slow growth rate of hair. In an attempt to overcome this limitation, we utilized transgenic mice in which a gene for a hair-specific marker, an ultra high sulphur keratin, is inserted to control a chloramphenicol acetyltransferase marker gene. Measurement of chloramphenicol acetyltransferase activity in hair or vibrissae follicles can then be followed as a sensitive measure of hair growth. The use of chloramphenicol acetyltransferase as a reporter for genomic events is a well-established and widely used method for measuring transfected gene activity in mammalian cells and transgenic animals $(1-3)$.

Due to the small amount of tissue available in single follicles, which would be optimal for assessing hair growth, a very sensitive assay is required. Previously, TLC-based radiometric and HPLC-UV detection methods have been used to detect chloramphenicol acetyltransferase activity in transgenic experiments, including hair growth studies. However, the analytic methods are not sufficiently sensitive to enable an accurate and quantitative assessment of chloramphenicol acetyltransferase activity in single follicles.

To achieve the necessary sensitivity in the chloramphenicol acetyltransferase assay for measuring enzyme activity in single follicles, we made use of a fluorescent chloramphenicol derivative as a substrate for the chloramphenicol acetyltransferase. When acetylated, the product is resolved by HPLC and the resulting fluorescence provides greater sensitivity than that previously reported using HPLC-UV detection methods. The analysis is also sufficiently sensitive for the easy detection of chloramphenicol acetyltransferase activity in single vibrassae follicles. This report describes the fluorescent assay and compares its sensitivity with that of an HPLC-UV detection-based assay.

\section{Materials and Methods}

The transgenic mice were offspring from a C57BI $\times$ B6sjc crossed with a CF1. The pups were 3 days old and weighed between 1.8 and 2.4 grams. These mice cxpress the chloram- 
phenicol acetyltransferase gene under the control of an ultrahigh sulphur protein gene promoter using the insert from a KER-CAT construct made by DNX Inc. (3). The ultrahigh sulphur protein gene expression was then quantitatively assayed in these follicles by the chloramphenicol acetyltransferase assay. The use of chloramphenicol acetyltransferase as a reporter for genomic events is a well established and widely-used method for measuring transfected gene activity in mammalian cells and in transgenic animals $(1-3)$. This enzyme has the advantage that it is prokaryotic, very stable, and a very sensitive marker (4). In previous reports we indicated that follicles from our transgenic mice can express chloramphenicol acetyltransferase as a marker for the ultrahigh sulphur protein gene (3). This chloramphenicol acetyltransferase gene expression is an excellent marker for hair formation since it is tissue specific and developmentally regulated and has been localized exclusively to the hair cortex by in situ hybridization (5). Procedures for whisker dissection have also been previously reported (6). The follicles were surgically removed and cultured in Dulbeccos Modified Eagle's Medium supplemented with a volume fraction of 0.20 fetal calf serum (Gibco, Grand Island New York).

\section{UV-HPLC chloramphenicol acetyltransferase assay}

Individual follicles were disrupted by ultrasonication for $4 \mathrm{~s}$ in $200 \mu \mathrm{l}$ Tris buffer $250 \mathrm{mmol} / \mathrm{l}, \mathrm{pH} 7.8$. Using a small $0.5 \mathrm{ml}$ polypropylene centrifuge tube, individual follicles were assayed in the same tube in which they were sonicated. To each tube, $20 \mu \mathrm{l}$ of $4 \mathrm{mmol} / \mathrm{l}$ acetyl coenzyme $A$, and $20 \mu \mathrm{l}$ of $30 \mu \mathrm{mol} / \mathrm{l}$ chloramphenicol (Boehringer Biochemicals, Indianapolis IN) were added. The assay was then incubated for up to $2 \mathrm{~h}$ at $37^{\circ} \mathrm{C}$. The reaction was stopped by the addition of $200 \mu \mathrm{l}$ ethyl acctate. The chloramphenicol and the acetylated products were extracted with ethyl acetate. The ethyl acetate was added to a micro sample tube (Sun Brokers, NC) and evaporated to dryness under vacuum. The dried, uncapped sample was placed in a sample vial (Kimbal Owens, IL) which was then placed in the autosampler rack. The Hitachi AS4000 autosampler was programmed to resuspend the sample in $55 \mu$ HPLC buffer just prior to the injection. Using the following HPLC conditions, $50 \mu \mathrm{l}$ were injected; buffer A: $1 \mathrm{ml} / \mathrm{l}$ trifluoroacetic acid (Pierce, Rockford, IL) in water; buffer B: $1 \mathrm{ml} / 1$ trifluoroacetic acid in acetonitrile (Burdick \& Jackson, Muskegon, MI); isocratic elution with $25 \%$ buffer B, flow rate $2.0 \mathrm{ml} / \mathrm{min}$ with a HS-3 C18 $3 \mathrm{~cm}, 3 \mu \mathrm{m}$ cartridge column (Perkin-Elmer, Norwalk, CA). The buffer conditions were maintained by the 8800 010 Ternary Solvent System (Spectra-Physics, San Jose, CA). The UV absorbance was monitored at $278 \mathrm{~nm}$ with a SpectraPhysics 8490-010 Programmable UV-Vis Detector and quantitated using the Spectra-Physics 2/80 Chromstation System Manager. The individual sample runs were stored and processed using the Spectra Physics 4270-310 Computing Integrator and down-loaded into a WIN 386 PC computer. For each sample the amount of chloramphenicol and acetylated chloramphenicol were calculated by peak area. Assay results were summarized as the area of individual peaks and as percent acetylation. Percent acetylation is equal to the sum of the 1-acetyl and 3acetyl chloramphenicol areas divided by the total areas of chloramphenicol, 1-acetyl and 3-acetyl chloramphenicol multiplied by 100 .

Fluorescent-HPLC chloramphenicol acetyltransferase assay

Individual follicles were prepared as in the UV procedure. To the Eppendorf tube, $20 \mu \mathrm{l}$ of $4 \mathrm{mmol} / \mathrm{l}$ acetyl coenzyme $\mathrm{A}$, and $0.5 \mu \mathrm{l}$ of $1 \mathrm{mmol} / 1$ Bodipy ${ }^{\mathrm{TM}}$ chloramphenicol (Molecular Probes Inc. Eugene, OR) was added. The assay was processed as in the UV method. The ethyl acetate was added to a sample vial (Kimbal Owens, IL) and evaporated to dryness under vacuum. The dried, uncapped sample was placed in the autosampler rack. The Hitachi AS4000 autosampler was programmed to resuspend the sample in $300 \mu \mathrm{l}$ HPLC buffer just prior to the injection. Using the following HPLC conditions, $50 \mu$ l were injected, buffer A: $1 \mathrm{ml} / \mathrm{l}$ trifluoroacetic acid (Pierce, Rockford, IL) in water; Buffer B: $1 \mathrm{ml} / 1$ trifluoroacetic acid in acetonitrile (Burdick \& Jackson, Muskegon, MI); isocratic elution with $27 \%$ buffer B, flow rate $2.0 \mathrm{ml} / \mathrm{min}^{\prime}$ with a HS-3 $\mathrm{C} 183 \mathrm{~cm}$, $3 \mu \mathrm{m}$ cartridge column (Perkin-Elmer, Norwalk, CT). The HPLC conditions were as in the UV method with the exception of the detector. The fluorescent emission was monitored at 512 $\mathrm{nm}$ and excitation was at $490 \mathrm{~nm}$ using the LC 240 fluorescence detector (Perkin Elmer, Norwalk, CT).

\section{Results}

Table 1 shows results for the detection of a purified standard of chloramphenicol by UV absorbance and the fluorescent derivative by fluorescence measurement. For each concentration the peak area was determined using the mean of five injections. In this comparative study, the lowest chloramphenicol concentration tested for UV detection was $5 \times 10^{-9} \mathrm{~mol}$; with fluorescence detection, a comparable peak area was obtained with $5 \times 10^{-12} \mathrm{~mol}$ of the fluorescent derivative. Thus the fluorescence detection is 1000 times more sensitive than UV detection. Identical concentrations of chloramphenicol and the fluorescent derivative cannot easily be compared for the two detection methods, since at concentrations where it was detectable by UV the fluorescence emission was almost off-scale. Where the fluorescence was on scale, the UV detection was at the low end of the detection limits. From this table we estimated the sensitivity limit for chloramphenicol using UV or fluorescence detections as $100 \mathrm{pmol}$ for UV and $100 \mathrm{fmol}$ for the fluorescence method. The standards were tested for linearity and both detection methods had a coefficient of greater than 0.95 .

Tab. 1. Comparison of sensitivity for the detection of chloramphenicol using UV absorbance and the detection of the fluorescent derivative using fluorescence emission. The fluorescence detection is 1000 -fold more sensitive than UV absorbance. Both methods are linear throughout the range of concentrations tested.

\begin{tabular}{lll}
\hline Detection method & $\begin{array}{l}\text { Chloramphenicol } \\
\text { injected } \\
(\mathrm{mol})\end{array}$ & Peak area \\
\hline UV & $1 \times 10^{-6}$ & $1.7 \times 10^{7}$ \\
& $5 \times 10^{-7}$ & $6.5 \times 10^{6}$ \\
& $5 \times 10^{-8}$ & $4.7 \times 10^{5}$ \\
& $5 \times 10^{-9}$ & $6.1 \times 10^{4}$ \\
Fluorescence & $5 \times 10^{-9}$ & $3.5 \times 10^{7}$ \\
& $5 \times 10^{-10}$ & $3.2 \times 10^{6}$ \\
& $5 \times 10^{-11}$ & $3.6 \times 10^{5}$ \\
& $5 \times 10^{-12}$ & $4.5 \times 10^{4}$ \\
\hline
\end{tabular}


Figure 1 shows the direct comparison between the UV and fluorescent detection methods on the same sample of the fluorescently-tagged chloramphenicol using both detectors at the same time. The chloramphenicol at $1 \times 10^{-9} \mathrm{~mol}$ gave a small detectable peak
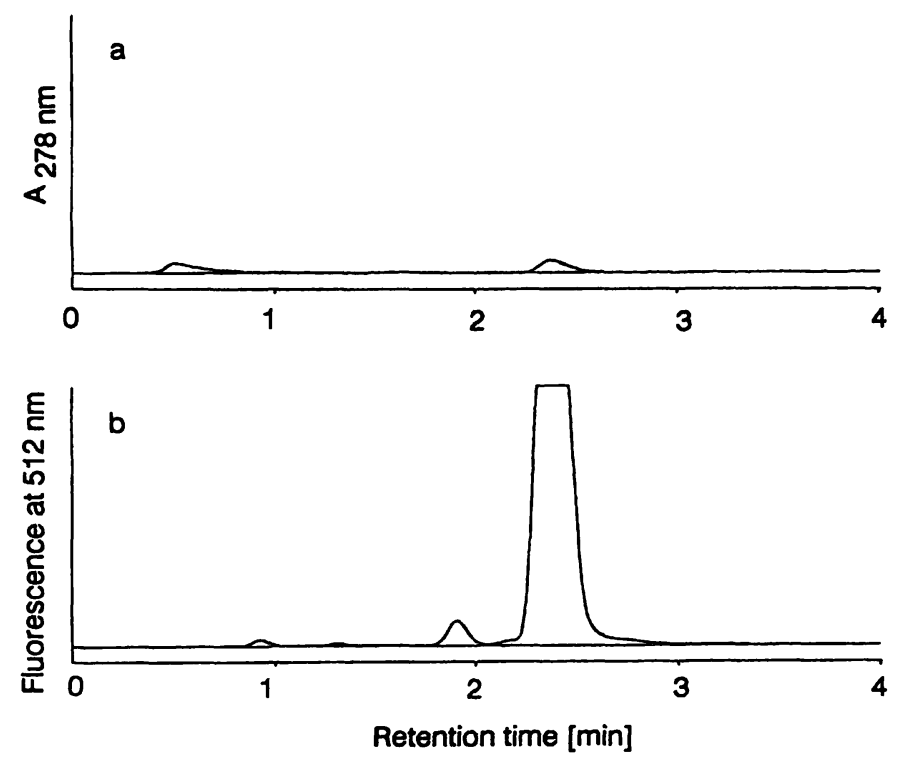

Fig. 1. Chromatograms of chloramphenicol detected by UV (a) or by fluorescence (b) of the same sample. To help visualize the peak detected by $U V$, and compensate for the difference in sensitivity of detection, the chromatogram peak shown in (a) has been increased 100 fold over (b). The area under the curve for chloramphenicol was 7001; whereas for $b$ the area under the curve was $26.7 \times 10^{6}$.
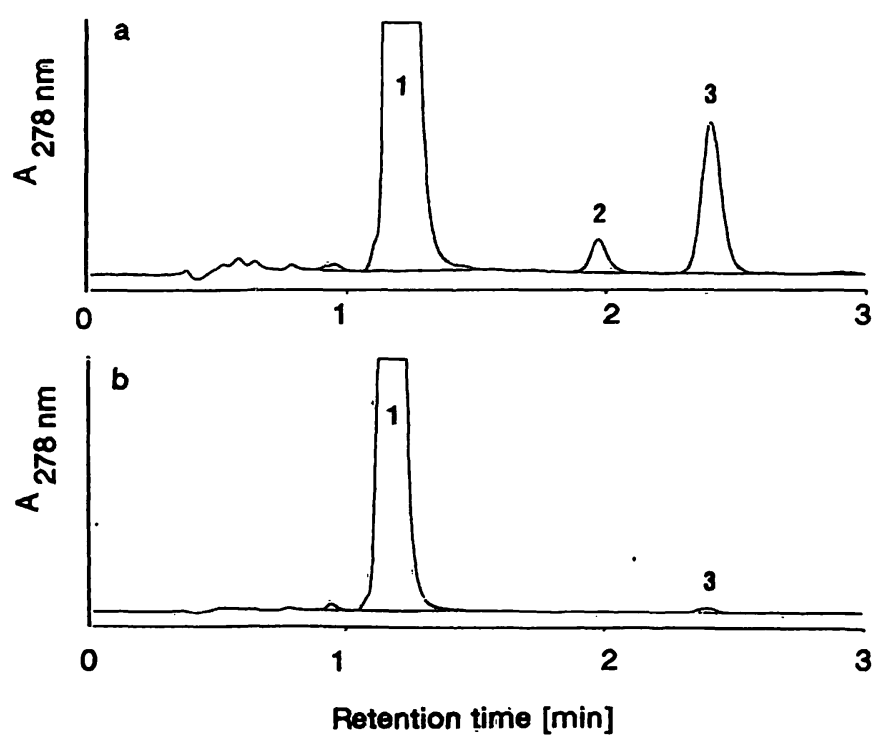

of 7001 by UV (A) but the fluorescence detection (b) produced a peak of $2.7 \times 10^{7}$. The chromatograms show the peak size for the fluorescent detection as larger than the UV peak. It should be noted that the chromatogram showing the peak for UV absorbance was amplified 100 fold over the fluorescent chromatogram to help visualize the peak.

Figure 2 shows the results for a chloramphenicol acetyltransferase assay using an extract from a representative fresh whisker follicle ( $a$ and $c$ ) and a whisker follicle after $18 \mathrm{~h}$ in culture (b and d). Both detection methods easily showed chloramphenicol acetyltransferase activity in the fresh follicle. UV detection is shown in $a$ and $b$; fluorescence detection is shown in $\mathrm{c}$ and $\mathrm{d}$. It should be noted that the UV method uses $100 \%$ of the sample whereas the fluorescent assay uses only $17 \%$ of the sample. The activity for the cultured follicle was less than that of the fresh follicle and sometimes fell below the UV detectable range. The small amount of acetylated chloramphenicol formed by the chloramphenicol acetyltransferase in these cultured follicles was still clearly detectable by the fluorescence method. The gene activity of ultrahigh sulphur protein was decreased in these cultured follicles but with the fluorescence detection method we were still able to assay the gene activity. To keep the run times to less than five minutes, the acetylated products of the fluorescent chlor-
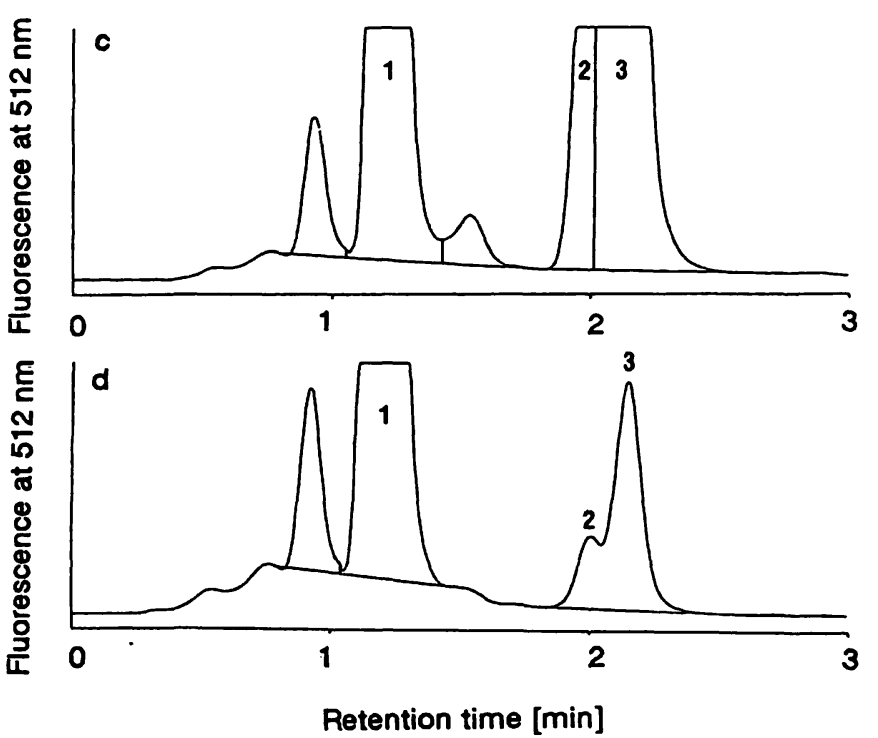

Fig. 2. Chloramphenicol acetyltransferase activity in a freshly dissected or cultured follicle, measured by UV or fluorescence detection.
a) UV detection: freshly dissected follicle
b) UV detection: cultured follicle
c) Fluorescence detection: freshly dissected follicle
d) Fluorescence detection: cultured follicle
Peak 1 is chloramphenicol
Peak 2 is 1 -acetyl chloramphenicol
Peak 3 is 3-acetyl chloramphenicol 


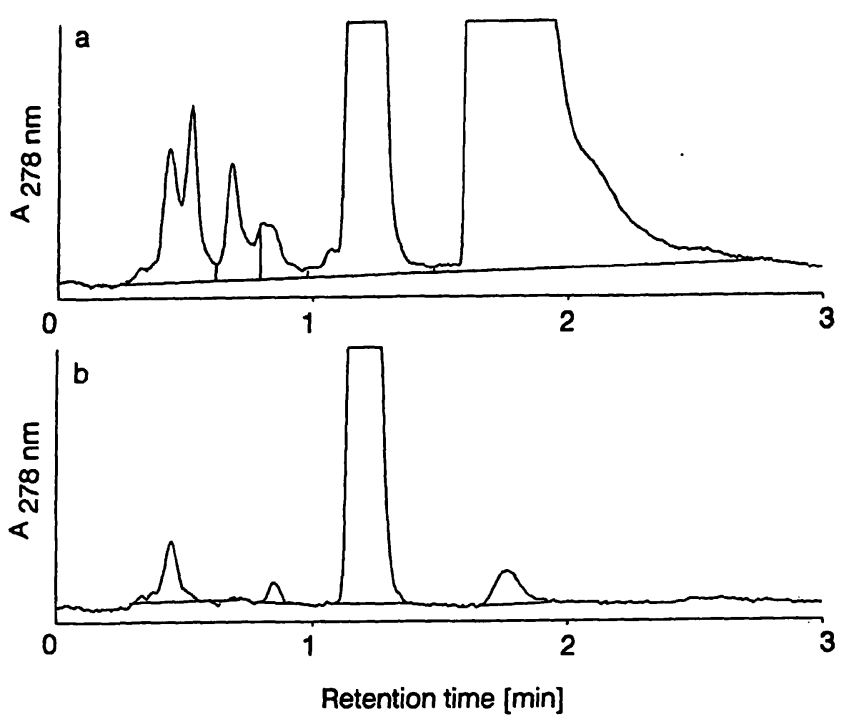

Fig. 3. The chromatograms show the false detection of chloramphenicol acetyltransferase activity. Chloramphenicol is detected at retention time $1.2 \mathrm{~min}$ and acetyl chloramphenicol at retention time $1.8 \mathrm{~min}$. Due to the nonspecific nature or UV detection, pinacidil was identified by retention time as acetylated chloramphenicol shown in a. The result of extensive washing to remove the pinacidil is shown in $b$.

amphenicol were not completely separated into two peaks, as in the UV method.

Figure 3 shows the advantage of the specificity of the fluorescence method over UV when dealing with unknown compounds. The first chromatogram was from a follicle treated with pinacidil (a compound known to cause hypertrichosis) (7) and then assayed for chloramphenicol acetyltransferase. Gene activity was determined by the presence of the acetylated peak at $1.68 \mathrm{~min}$ retention time using the UV detection method. Pinacidil was added to the culture media of a follicle in which the ultrahigh sulphur protein was to be assayed. This compound appears to have increased the amount of ultrahigh sulphur protein gene activity in the follicle, because a large peak appears where acetylated chloramphenicol should be. Closer inspection revealed that the substrate peak was not diminished and the suspected product peak was larger than the initial substrate concentration used. This indicated that the peak at 1.68 min retention time was not mono-acetylated chloramphenicol. Further investigation revealed that the peak was, in fact, pinacidil. Extensive rinsing of the follicle gave the result shown in panel $b$, which shows that the original peak was not acetylated chloramphenicol but an artifact of pinacidil being carried over in the follicle tissue. Pinacidil was detected by UV absorbance and proved to be a false positive by co-migrating with the monoacetyl chloramphenicol. Pinacidil is not fluorescent and cannot be detected by fluorescence emission. This false positive was revealed by careful data analysis, but indicated the problem of the non-selectivity of the UV detection method.

$\cdot 1$

\section{Discussion}

These results show that the sensitivity of chloramphenicol acetyltransferase activity detection can be increased by using a fluorescently-tagged chloramphenicol. Detection of Bodipy ${ }^{\mathrm{TM}}$ chloramphenicol by peak area gives as much as a 5000 fold increase over the peak area detected by UV absorbance. With this increased sensitivity, the fluorescent method has a greater base line fluctuation at low attenuations than the UV method. This limits the sensitivity for very small samples but since the detection sensitivity increases more than the base line fluctuation, there is an overall 1000 -fold increase in the detection of chloramphenicol using the fluorescent method. This increased sensitivity of the assay represents a substantial improvement in chloramphenicol acetyltransferase assay technology. With this assay procedure the chloramphenicol acetyltransferase assay is now fast, applicable to large numbers of assays, specific for genomic events, requires no radioactive material, and is more sensitive than UV-HPLC assays. This HPLC fluorescent chloramphenicol acetyltransferase assay will be a useful tool for the study of hair biology, since it enables the rapid assay of hair-specific gene regulation.

We found that the fluorescence was degraded in the TRIS buffer. Samples left overnight in buffer showed a significant loss of activity. Addition of the buffer just prior to injection eliminated this problem.

Additionally, when we compared the acetylation of chloramphenicol with that of the fluorescently-tagged chloramphenicol, the tagged chloramphenicol showed a higher percentage of acetylation. This may indicate that the reaction conditions favour the fluorescent chloramphenicol substrate, or that chloramphenicol acetyltransferase is inherently more active towards the tagged substrate.

One disadvantage in using the fluorescent assay method compared with the UV detection method is that the mono-acetylated chloramphenicols cannot easily be totally separated from each other with this procedure, whereas the UV method can separate the 1-acetyl from the 3-acetyl chloramphenicol. A longer column would separate the two mono-acetylated products but this was not required for our analysis, since the two areas would simply be recombined in order to calculate total products. 


\section{References}

1. Burzio, L. O., Brito, M., Zarrage, A. M. \& Siddiqui, M. A. (1988) Assay of Chloramphenicol Acetyl Transferase by High-Performance Liquid Chromatography. Gene Anal. Techn. 5, 5-8.

2. Young, S. L., Jackson, A. E., Puett, D. \& Melner, M. H. (1985) Laboratory Methods: Detection of Chloramphenicol Acetyl Transferase Activity in Transfected Cells: A Rapid and Sensitive HPLC-based Method. DNA 4, 469-475.

3. McNab, A. R., Andrus, P., Buhl, A. E., Waldon, D. J., Kawảbe, T. T., Rea, T. J., Groppi, V. \& Vogeli, G. (1990) Hair Specific Expression in Transgenic Mice of Chloramphenicol Acetyl Transferase Under the Control of the Ultra High Sulfur Keratin Promoter. Proc. Natl. Acad. Sci. USA $87,6848-8652$.

4. Gorman, C. M., Moffat, L. F. \& Howard, B. H. (1982) Recombinant Genomes Which Express Chloramphenicol Acetyltransferase in Mammalian Cells. Mol. Cell. Biol. 2, 1044-1051.
5. Vogeli, G., Wood, L., McNab, A. R., Kaytes, P., Wagner, T. E., Tea, T. J., Groppi, V., Waldon, D. J., Kawabe, T. T. \& Buhl, A. E. (1991) High-Sulfur Protein Gene Expression in a Transgenic Mouse. Ann. New York Acad. Sci. 642, $21-31$.

6. Buhl, A. E., Waldon, D. J., Kawabe, T. \& Holland, J. M. (1989) Minoxidil Stimulates Mouse Vibrissac Follicles in Organ Culture. J. Invest. Dermatol. 92, 315-320.

7. Buhl, A. E., Waldon, D. J., Conrad, S. J., Mulholland, M. J. Shull, K. L., Kubicek, M. F., Johnson, G. A., Kamdar, B. V., Thomasco, L. M., Schostarez, H. J. \& Schwartz, T. M. (1992) Potassium Channel Conductance: A Mechanism Affecting Hair Growth Both In Vitro and In Vivo. J. Invest. Dermatol. 90, 315-319.

Daniel J. Waldon

The Upjohn Company

9157-25-12

Kalamazoo, MI 49007

USA 
